Revista Venezolana de Gerencia (RVG)

Año 16. $\mathrm{N}^{\circ}$ 56, 2011, 595 - 607

Universidad del Zulia (LUZ) • ISSN 1315-9984

\title{
Categorías de la acción gerencial universitaria autónoma
}

\author{
Rondón García, Yoselina*
}

\section{Resumen}

Esta investigación tiene como finalidad generar categorías para redefinir los estilos de acción gerencial en la educación superior venezolana. El escenario donde fue focalizada la investigación fue la Universidad de Oriente en sus cinco núcleos, considerando como informantes clave a profesores, estudiantes y empresarios de la zona. La investigación es de tipo cualitativo. Para el análisis de los datos y la generación de categorías se utilizó la teoría fundamentada de Strauss y Corbin (2002). Posteriormente, se llegó a una reflexión final en la que se consideró la necesidad del desarrollo de una gerencia sustentada en pilares axiológicos, praxiológicos, epistemológicos y teleológicos que permitan redimensionar la visión y misión de estas instituciones y adaptarlos a estilos gerenciales más colaborativos y sensibles ante los procesos de transformación.

Palabras clave: Gerencia universitaria, educación superior, categorías, teoría fundamentada.

\section{Categories of Managerial Action in the Autonomous University}

\begin{abstract}
This research aims to generate categories for redefining management action styles in Venezuelan higher education. Research took place at the five campuses of the Universidad de Oriente (University of the East). Key informants were teachers, students and entrepreneurs in the area. The research was qualitative. For analysis, the grounded theory of Strauss and Corbin (2002) was used. The results were categories that emerged from the key informants' discourse about university managerial action. Later, a final reflection considered the need to develop management supported by axiological, praxeological, epistemological and teleological pillars that make it possible to re-design the vision and mission of these institutions and adapt them to more collaborative and sensitive managerial styles in the light of transformation processes.
\end{abstract}

Key words: University management, categories, redefinition, collaborative.

Recibido: 20-04-11 Aceptado: 09-11-11

* Doctora en Gerencia. Profesora Asociada a dedicación exclusiva de la Escuela de Ciencias Administrativas de la Universidad de Oriente, Núcleo Anzoátegui. Investigadora Activa. Premio de Estímulo al Investigador (PEI, 2011). e-mail: yoselina1@hotmail.com 
Categorias de la acción gerencial universitaria autónoma

Rondón García, Yoselina

\section{Introducción}

La necesidad de reivindicación de valores en todos los niveles de la educación superior (léase directivos, profesores, estudiantes, personal administrativo y obrero) juega un papel muy importante en estos procesos de transformación que abarca las dimensiones sociales, políticas y económicas del país. Este factor se perfila como el ingrediente indispensable para producir verdaderos cambios en el quehacer universitario.

En la Declaración de la Conferencia Regional de la Educación Superior en América Latina y el Caribe (CRES, 2008). Se resaltaron aspectos importantes que incitan a reflexionar sobre la reivindicación de valores altruistas en el quehacer universitario los cuales son un punto clave en el repensamiento de la identidad universitaria como vía para generar compromisos genuinos por parte de la comunidad estudiantil, profesoral y personal administrativo y obrero que permitan acelerar los procesos de transformación.

En un mundo globalizado que, a pesar de los avances tecnológicos cada vez más significativos, es socialmente injusto y desigual, hay que consolidar el papel de la Educación Superior (ES) en la región como un factor estratégico para el desarrollo sustentable y para la promoción de la inclusión social y la solidaridad regional, y para garantizar la igualdad de oportunidades. En este contexto, las Instituciones de Educación Superior (IES), en el ejercicio de su autonomía, deben asumir un claro compromiso social y su responsabilidad como servicio público, promoviendo el desarrollo de la ciencia y la tecnología, al mismo tiempo que pro- gramas y actividades constructoras de los aspectos humanísticos y que apunten a la formación integral y de ciudadanía.

El Estado en sintonía con esta necesidad de transformación ha generado políticas y planes orientados a la necesidad de refundar los valores y crear una sociedad venezolana más humana, es por ello que el Ministerio de Planificación y Desarrollo (2007:5), presentó un documento titulado: Plan de Desarrollo Económico y Social de la Nación 2007-2013, en el cual expone lo siguiente "La plena realización del Socialismo del Siglo XXI que estamos inventando y que sólo será posible en el mediano tiempo histórico, pasa necesariamente por la refundación ética y moral de la Nación venezolana...."

Lo que se deduce de la cita anterior es que los procesos de transformación, reivindicación de valores, y mejoramiento de la praxis gerencial debe ser considerada como un compromiso colectivo, que requiere de la participación de todos los entes involucrados en ese proceso. Esta necesidad hizo que creciera el interés de la investigadora en avanzar en el estudio de la gerencia universitaria como ente clave para liderizar los procesos de transformación requeridos por la nación, y por ende, por las instituciones de educación superior, por ser estas organizaciones las que tienen en su seno la responsabilidad de formar los futuros profesionales que van a construir el cambio que requiere la República.

La educación tiene siempre una mirada prospectiva, la de construir el futuro. Además de atender a problemas inmediatos y prácticos, la educación superior tiene el cometido de preservar las conquistas de la historia humana en los campos del co- 
nocimiento, del pensamiento, de las realizaciones artísticas y culturales, y, asimismo, anticiparse a la sociedad de su tiempo y buscar construir un futuro más acorde con los principios y valores que edifican la humanidad. Si el presente está lleno de incertidumbres, y si tantos y acelerados son los cambios de diversa naturaleza, una grave cuestión se instala: ¿qué futuro deseamos, podemos y necesitamos construir? En gran parte, el futuro de la educación superior depende de las respuestas que hoy demos a esa pregunta, del compromiso de sus principales actores (Directivos, docentes, estudiantes, comunidades), y de la conciencia histórica que se tenga de sus luchas.

En este artículo, se hace un análisis sustentado en la revisión de teóricos expertos en el área de gerencia los cuales hacen referencia al paradigma rígido, burócrata e individualista que ha caracterizado la praxis gerencial universitaria en Venezuela, y proponen mecanismos a través de los cuales se puede transformar estas prácticas gerenciales a estilos más colaborativos. Posteriormente, sustentándose en estas referencias teóricas se procedió a indagar a través de informantes claves (profesores, alumnos, empresarios) su percepción sobre como redefinir los estilos gerenciales universitarios en Venezuela.

El tipo de investigación es de caracter cualitativo, lo que permitió que el diseño de la misma, sea flexible y de enfoque progresivo, sensible a los cambios y modificaciones de acuerdo con las circunstancias que la rodeen. Como método se precisó de una matriz de comprensión basada en la hermenéutica, donde se resaltó las estrategias de este método, el cual permitió construir un mapa de categorías, visiones y perspectivas de los actores seleccionados que se contrastaron con investigaciones referenciales con el fin de generar elementos para definir categorías que permitan construir una nueva concepción de la acción gerencial universitaria autónoma.

El escenario en el cual se desarrolló la investigación estuvo constituido por los cinco núcleos de la Universidad de Oriente (Anzoátegui, Monagas, Sucre, Nueva Esparta, Bolívar). Los grupos de actores se seleccionaron dada la importancia de sus roles y representaciones discursivas respecto al contexto universitario (profesores, alumnos y empresarios), y de interés particular de la investigadora, por encontrase inmersa en el área docente y de investigación universitaria; tomando la opción ontológica asumida por la metodología cualitativa que exige que la muestra no puede estar constituida por efectos aleatorios escogidos al azar y descontextualizados (Martínez, 2004).

La elección de informantes se realizó tomando en cuenta los siguientes criterios:

Accesibilidad: Se focaliza la Universidad de Oriente por ser el escenario en el cual se desenvuelven la investigadora (La investigación se focalizará en los núcleos de Anzoátegui, Monagas, Sucre y Nueva Esparta de la Universidad de Oriente).

Construcción del Perfil del informante: Los informantes se seleccionaron en función de las siguientes características:

1. Profesores: Especializados en el área de Administración y Gerencia (10 profesores), con no menos de 18 años laborando en la institución (no excluyen- 
Categorias de la acción gerencial universitaria autónoma

Rondón García, Yoselina

te), con al menos 1 año experiencias en cargos gerenciales universitarios (jefes de Departamentos o Coordinadores Administrativos y/o académicos) y/o alguna experiencia en gerencia en empresas privadas (no obligatorio). La justificación de la selección de este perfil responde a la necesidad que implica el tipo de investigación respecto a consultar docentes que exhiban un perfil sólido y complementario en lo gerencial y académico.

2. Estudiantes: Se aplicó la técnica de grupos focales de discusión a estudiantes en proceso de grado, de la línea de investigación "Gerencia y Transformación Universitaria" (20 estudiantes), en la Escuela de Ciencias Administrativas del Núcleo de Anzoátegui. Este grupo tuvo la oportunidad de reflexionar por tres meses sobre el tema de gerencia universitaria, políticas públicas en materia de educación superior y tendencias de la gerencia. Luego de estas reflexiones se les aplicó la técnica antes señalada para que emitieran sus percepciones sobre el objeto de la investigación y aporten conclusiones grupales.

3. Empresarios de la zona oriental: Se seleccionaron 8 empresarios en esta área, cuyo criterio incluyente fue que el tiempo de experiencia fuese de 10-15 años en el área gerencial, pertenecientes a empresas reconocidas de la zona, y que hayan egresado y sean empleadores de profesionales de la Universidad de Oriente.

Estos informantes claves corresponden a un primer avance de esta investigación, se estima extender posteriormente el estudio a otros actores de la comunidad (administración pública, consejos comunales, entre otros.)
Para hacer referencia al discurso vivo de estos informantes en el desarrollo de esta investigación cada actor clave se designó de la siguiente forma: Profesores (Prof), Estudiantes (Est), Empresarios (Emp).

En cuanto a la técnica y criterio en la recogida de información, se trabajo con 1. la entrevista semi-estructurada, la cual es guiada por un conjunto de preguntas y aspectos básicos a explorar, pero ni la redacción exacta ni el orden de las preguntas que se construyen está predeterminado (Valles, 1999). Estas entrevistas fueron aplicadas a profesores y empresarios de la zona según criterios de selección y segmentación expuestos anteriormente, $y, 2$. La técnica de grupos focales, en esta técnica hay que asegurarse que los participantes tengan una experiencia específica u opinión sobre la temática o hecho de investigación; requiere de un guión de funcionamiento que reúna los principales tópicos a desarrollar. El escenario donde se realizó la actividad fue la Sala de Conferencias de la Escuela de Ciencias Administrativas de la Universidad de Oriente Núcleo de Anzoátegui, y fue aplicada al grupo de estudiantes según los criterios de selección y segmentación expuestos anteriormente.

Las entrevistas semi-estructuradas fueron grabadas para obtener de los discursos vivos de los informantes, las percepciones que permitan elaborar la construcción social derivada del objeto de estudio.

La técnica de grupos focales, fueron grabadas y resumida por los participantes en papeles de trabajo.

La interpretación del material, se realizó congruentemente con la postura 
epistemológica y el método asumido en la investigación (paradigma interpretativocomprensivo - investigación cualitativa método hermenéutico). Además se utilizó como referente para el análisis e interpretación de la investigación la Teoría Fundamentada de Strauss y Corbin (2002). Esta teoría permitió descubrir desde los registros, grupos analíticos de datos, conformado por la construcción de lo que Strauss y Corbin llamaron códigos, categorías y memos.

\section{Referentes teóricos sobre la acción gerencial universitaria}

Tapscott (1998) hace una reflexión considerando que la nueva economía es del conocimiento y el aprendizaje, por tanto las instituciones universitarias que no se reinventen a sí mismas para responder a los cambios que plantea el entorno por tradiciones educativas que datan de siglos atrás o por resistencia al cambio, verán desplazada la misión que les corresponde como entes formadores hacia otros ámbitos, como por ejemplo las empresas y/o instituciones educativas paralelas, que desarrollen redes interactivas de educación multidisciplinaria para atender las necesidades de la sociedad post-moderna. Este panorama, según Castells (2009), reclama una gestión más activa y atenta a la competencia por prestigio, por recursos y por legitimidad, la nueva gerencia universitaria debe demandar estructuras más abiertas, con mayores compromisos de colaboración, y menos burócratas.

Morín (1999), en su enfoque de la complejidad hace un planteamiento inte- resante respecto al cambio que se debe generar en las concepciones paradigmáticas que tienen los principales actores de la universidad y sostiene que la verdadera reforma educativa, requiere un profundo cambio en los esquemas tradicionales de gerenciarla. Pero para que este tipo de reforma sea viable se necesita remover los cimientos más profundos de la naturaleza humana.

Otro trabajo reciente corresponde a la investigación de Muros (2004), la cual a través de un estudio cualitativo cuya fuente de información fueron actores claves en el área de la gerencia universitaria (expertos y pensadores en el área de reforma universitaria y representantes de instituciones rectoras de educación superior), expone que las construcciones discursivas de los gerentes de las universidades convergen en la necesidad de nuevas formas de dirigir el mundo universitario, y en la necesidad de humanizar la gerencia y rescatar el contenido de la condición humana en contrapartida al personalismo y el egocentrismo; Para esto, es necesario lograr la madurez ética, intelectual y política de las autoridades universitarias.

La autora en referencia afirma que en la gerencia universitaria persisten construcciones discursivas, políticas e ideológicas propias de la modernidad. Desde la racionalidad técnico-instrumental presente en la lógica de los discursos analizados se manifiestan mecanismos de control cargados de la noción de lo que es verdadero, de lo que es posible conocer y de los sujetos que son capaces de dar cuenta de la definición de la problemática de la gerencia universitaria. Esta reflexión de Muros, abre marcos de comprensión respecto a como los gerentes 
Categorías de la acción gerencial universitaria autónoma

Rondón García, Yoselina

universitarios (entrevistados en su investigación) desde su racionalidad técnicoinstrumental, excluye la necesidad de la participación de otros actores en la discusión de la transformación universitaria, el marcado énfasis en la eficiencia-productividad-resultado, dibuja un entendimiento del deber ser del gerente enmarcado dentro del cumplimiento de sus funciones, y demuestra una carente visión estratégica.

Por otra parte, Méndez (2004) expone que, las propuestas de transformación en el ámbito universitario son abundantes, ya que en diversas épocas ha existido una tendencia bastante fuerte respecto a la transformación de la universidad. Dentro de esta serie de propuestas, el autor hace mención a los trabajos que viene realizando por más de una década la UNESCO, OAL y el IESAL para estudiar la educación superior en América Latina. Por ejemplo la UNESCO, propone tres principios básicos que los colocan como punto de honor para la transformación de todas las estructuras universitarias: pertinencia, calidad e internacionalización de la Educación Superior.

Este plan condujo a muchas iniciativas desde 1990. Sin embargo los resultados no pasaron más allá de ser enunciados. Según el autor, esta inaplicabilidad de las propuestas, responde a que la UNESCO, nunca hizo un diagnóstico que explique mediante una teoría científicointegral la realidad universitaria en el contexto histórico-latinoamericano-global, ya que esta organización por motivos de diplomacia no se involucra en las especificidades de cada país o región.

Méndez (2004: 47), reflexiona respeto a estos puntos y destaca: "que lo que genera esta inoperancia en la puesta en práctica de las propuestas de transformación, radica en una crisis paradigmática que toca los fundamentos del conocimiento y de la ciencia en particular, de la concepción del hombre que se tiene y que se quiere formar, a los fundamentos de la sociedad que tenemos y queremos construir. Por lo tanto hay que desmontar las bases epistemológicas desde la cual se produce el conocimiento en la ecología universitaria y difundir una nueva concepción de la praxis universitaria que responda a los escenarios actuales". Pero para lograr esta transformación el autor afirma que la transformación no puede pensarse destruyendo lo existente. $\mathrm{Ni}$ puede ser impuesta. Debe hacerse científicamente, utilizando las herramientas gerenciales existentes que conciben la transformación y el cambio sin paralizar la organización, utilizando planes a largo plazo y acometiendo estrategias progresivas de cambio a corto y mediano plazo. Y contando sobre todo con el consenso interno de la propia comunidad universitaria y con el apoyo estratégico de las instituciones del medio ambiente.

Otro aporte respecto a este tema es el de Fergusson (2003), el cual expone que la responsabilidad de la universidad está en crisis, por ende sus estructuras organizativas y políticas están en dudas. Al igual que el paradigma de producción de conocimiento que la permea, ha perdido vigencia y ha afectado la producción de saberes. Fergusson afirma que es necesario comprometer los esfuerzos en realizar cambios radicales en la política académica universitaria, ya que de lo contrario se seguirá reproduciendo la pedagogía de la domesticación, alimentando una visión 
instrumental de la naturaleza y el mundo, y reproduciendo un modo de vida cuya lógica de la rentabilidad económica, subordina el interés colectivo por el mejoramiento de la calidad de vida humana y la salud ambiental a los intereses individuales o de los grupos económicos y políticos que controlan el poder.

Por lo tanto este autor propone que se debe producir un movimiento de actores, instituciones y comunidades en una dinámica que optimice la complejidad como problemática en la educación superior. La cooperación permite amplificar hoy día, en un universo plural y mundializado, el enraizamiento cultural y la innovación científica.

El éxito en la transformación de la gerencia universitaria converge con un abanico de perspectivas relacionadas con la gestión y la participación. Llanos, (1997:227), en un estudio referente a la gestión de las universidades nacionales, afirma que "el éxito, debe atender a los principios de planificación estratégica participativa. Llanos, hace una revisión de cinco modelos de gestión exitosa para las universidades nacionales: modelo recurso-sistema; modelo efectividad sobre calidad: modelo satisfacción participante; modelo de metas y el modelo contradicción de la efectividad". Este último modelo se considera a la universidad como: un sistema social abierto y a cada grupo que integra su comunidad y al entorno como subsistemas interactuantes que se relacionan con la universidad pero también reciben las influencias del entorno, los cuales les impone acciones de aceleración hacia delante o retroceso.

Las investigaciones antes expuestas, coinciden en darle importancia a la par- ticipación no solo de los gerentes universitarios, sino también a los actores que se encuentran en sus ejes de incidencia, a fin de llegar a un consenso más próximo a lo que debe significar la gerencia universitaria en el contexto específico donde se encuentra. Otro aspecto que cabe resaltar, es la necesidad de repensar la universidad y sus estilos de gerencia, esto implica una gran reflexión por parte de los gerentes y la comunidad intra y extra universitaria considerando que es necesario un debate epistemológico, como lo expone Lanz (2005), cuando precisa que si se logra recuperar la relevancia del debate epistemológico se ganará un valioso terreno para la recreación de los presupuestos de otras formas de pensamiento. Este filósofo investigador, afirma que es necesario explicitar hasta sus últimas consecuencias las bases epistemológicas sobre las que reposan los análisis de la universidad. Solo de esta matriz epistémica es que depende buena parte de lo que se propone en materia de reforma académica. En función de esta afirmación Lanz identifica vectores de acción para deconstruír las bases epistemológicas existentes, entre los más relevantes para esta investigación, se tienen: (a) Asumir la transcomplejidad como acción reflexiva para repensar la universidad y (b) Una apuesta fuerte para el diálogo de saberes y consenso entre seres humanos.

Las anteriores investigaciones abren un marco epistemológico interesante en el cual se matizan campos de reflexión, para esta investigación, ya que en la presente se pretende contribuir con la construcción teleológica-epistémico-axiológica-praxiológica para generar catego- 
Categorías de la acción gerencial universitaria autónoma

Rondón García, Yoselina

rías re-definitorias de la acción gerencial universitaria autónoma. Con esto se requiere considerar la gerencia universitaria como un fenómeno complejo y multidimensional cuya construcción, demanda de un auténtico consenso colectivo intra y extra universitario donde el interés individual se identifique con el interés del conjunto de actores claves, y de una referencia teórica crítica que permita fomentar los escenarios para la construcción dialógica que requiere este planteamiento.

\section{Proceso de análisis e interpretación de las categorías redefinitorias de la acción gerencial en la Universidad de Oriente}

A continuación se presenta el análisis e interpretación de los datos derivados de las distintas entrevistas realizadas a tres grupos de informantes claves: Profesores, alumnos, empresarios, considerando que la investigación persigue la generación de categorías desde los discursos de los informantes claves de la Universidad de Oriente y del ámbito empresarial oriental para proponer una re-definición de los estilos gerenciales universitarios enmarcados en su acción gerencial. A través del análisis e interpretación de los datos se etiquetaron una serie de párrafos en las entrevistas a cada informante lo cual originó categorías que dimensionan o catalogan el objeto de estudio.

Esta primera etapa de codificación abierta originó una serie de familias de códigos que orientan las categorías que se persiguen formular. La codificación abierta se realizó en función de un área temática clave, orientada a los aspectos axiológicos, praxiológicos, teleológicos y epistemológicos de lo que debería ser la gerencia universitaria.

Continuando con el análisis e interpretación de los datos, luego que se han codificado los diferentes elementos que emergieron de las entrevistas se formaron familias de códigos de las cuales surgieron cuatro categorías conceptuales las cuales se muestran a continuación:

\subsection{Aspectos axiológicos de la gerencia universitaria}

Los valores en términos generales pueden definirse como el convencimiento de determinadas creencias, acuerdos o principios y actúan como criterios para la toma de decisiones. La palabra valor tiene tres dimensiones, una dimensión económica, una dimensión ético-estratégica y una psicológica. Los valores individuales se constituyen y se aprenden fundamentalmente en la infancia y la adolescencia a partir de modelos sociales, padres, maestros y amigos en general del entorno familiar.

Por su parte, la Universidad en el marco de las organizaciones de educación superior construye comunicativamente acuerdos como estrategia de incorporación de actores, quienes comparten valores de compatibilidad (estudiantes-docentes-directivos), valores de construcción de un saber de integración, reconoce la diversidad y tolerancia, se descubre la complementariedad, todo ello para construir una vida mejor. La Universidad resulta un espacio cosmopolita para formar valores e incorporar nuevos conocimientos, habilidades, hábitos y ca- 
pacidades que preparan al egresado para la vida.

Desde la perspectiva de los informantes claves la gerencia universitaria debe ser ejercida en base a un conjunto de valores que han sido debidamente identificados y recogidos en el marco regulatorio de la educación superior venezolana y sus diferentes propuestas sobre transformación, en este mismo sentido uno de los informantes afirmó que:

Prof $8 . \ll$. Es imprescindible que en la universidad exista la solidaridad fundamentada por medio de normas y valores que deben surgir de una nueva moral que humanice la universidad y la integre al sistema social para que exista una misión consecuente con las necesidades que la sociedad reclama satisfacer.>>

Desde la perspectiva de varios de los entrevistados la identidad que tenga con la institución el gerente universitario es decisiva en los procesos de transformación ya que esto determina el compromiso genuino para establecer estrategias en pro del mejoramiento de los procesos institucionales. El sentido pluralista referido a la actitud de cooperación y solidaridad entre los miembros de la comunidad universitaria son valores vitales que deben caracterizar al líder universitario para aunar esfuerzos y comprometer a su comunidad en el logro de objetivos comunes. La transparencia o rendición de cuentas claras también es un dispositivo que genera confianza en el colectivo.

Otro aspecto señalado por los informantes claves es la necesidad de darle al aspecto político el lugar que le corresponde, es imprescindible resaltar a la academia sobre cualquier interés político que desvirtué la verdadera esencia del quehacer universitario.

Uno de los informantes claves acoto que el valor referido a la solidaridad tiene que existir en el ámbito universitario dentro de una nueva moral que integre el subsistema universidad al sistema social para que exista una misión consecuente con las necesidades que la sociedad reclama satisfacer

Se exponen elementos muy importantes sobre que la gerencia personal se debe proyectar hacia la gerencia institucional. Un estudiante entrevistado afirma lo siguiente al respecto: Est10. “...una persona que no sepa gerenciar su vida menos tendrá la capacidad para gerenciar eficientemente una organización que requiere de principios y valores bien arraigados".

Respecto al comportamiento ético un empresario entrevistado sostiene que Emp7. "El líder universitario debe caracterizarse por un comportamiento ético en su desempeño del día a día”.

\subsection{Aspectos teleológicos de la gerencia universitaria}

El sentido de servicio en el ejercicio de la gerencia, y la capacidad de amar lo que se hace fueron los aspectos más resaltantes en esta categoría. A este respecto uno de los informantes acoto lo siguiente: Est11. $<<$ Yo pienso que un gerente universitario (entiéndase rector, decano, director de escuela, jefe de departamento, coordinadores de comisión), ante todo debe amar a la universidad, ser un sacerdote de la misma, eso es algo trascendental que debe tener alguien que se dedique al área académica>>. 
Categorías de la acción gerencial universitaria autónoma

Rondón García, Yoselina

Varios de los informantes coincidieron en que es importante superar el individualismo que caracterizan a la sociedad contemporánea actual pero para lograr esto los cambios deben experimentarse desde lo profundo del individuo. A este respecto uno de nuestros informantes afirma: Emp5. $<<Y_{0}$ pienso que primeramente debe poner a la política en el lugar que le corresponde. Debe hacer énfasis en lo académico. Organizarse de una forma diferente, no por jerarquías, las instituciones educativas deben estar organizadas por grupos de trabajo, que sigan un proceso lógico de autogestión universitaria. Deben también repensar y reflexionar mucho sobre su función y crear nuevos mecanismos de comunicación con el estudiantado, crear objetivos comunes con el interés del estudiante, dejar de decidir para las elites.... Yo creo que allí esta la clave del éxito de los procesos gerenciales universitarios $>>$.

Se debe ejercer la gerencia con un sentido de trascendencia basado en el desapego material y afianzado en el servicio al otro como aspecto fundamental en la fijación de metas, visión y misión de la gerencia universitaria. Resulta imperativo humanizar la gerencia universitaria, rescatar la condición humana en contrapartida al personalismo, egocentrismo y demás desviaciones presentes. Hay que democratizar el manejo del poder como elemento necesario que permite el reconocimiento de la existencia del otro como ser social.

\subsection{Aspectos epistemológicos de la gerencia universitaria}

La visión de totalidad es una categoría clave para establecer nuevas for- mas de organización donde el reconocimiento de la complejidad permita a la alta dirección de las universidades darse cuenta de la lentitud de sus aprendizajes, de la ficcionalización de sus interpretaciones políticas y la miopía ante las transformaciones que deben impulsar. Los discursos vivos de los informantes coinciden en que se debe presentar una propuesta para el diseño de un programa de formación dirigido a los equipos gerenciales de Educación Superior.

A este respecto uno de los informantes agregó: Prof. $8<<$ Pienso que es muy importante formar cuadros gerenciales en las instituciones de educación superior, esto implicaría una serie de elementos formativos desde liderazgo hasta la gestión y utilización de recursos para el aprovechamiento de la academia. El desarrollo de una visión más holística y el reavivamiento de valores definitivamente requiere de procesos formativos $>$.

Según los informantes, entender que el mecanismo no son las jerarquías organizacionales sino los equipos de procesos, como formas planas y flexibles, que comparten el conocimiento y se apoyan a partir de un permanente aprendizaje colaborativo. En este mismo orden de ideas los informantes afirmaron que el sentido comunitario es vital para las organizaciones porque brinda una fuerte inspiración de compromiso e identificación de necesidades en las comunidades. Además es una fuente que propulsa el cambio paradigmático que se necesita para que puedan generarse transformaciones en los estilos gerenciales. 


\subsection{Aspectos praxiológicos de la gerencia universitaria}

Los aspectos que deben guiar la praxis del gerente universitario, según las perspectivas de los informantes, se fundamentan en abrir auténticos espacios para la participación democrática en los problemas fundamentales de la institución con propuestas creativas e innovadores que pueden surgir desde la base de la organización que se materialicen en tomas de decisiones resultantes de negociaciones y acuerdos. Además se deben revisar las estructuras y niveles jerárquicos, con el fin de lograr la flexibilización y lograr sistemas organizativos planos que permitan desplazar la práctica centralizada lo cual conduce al fortalecimiento de la participación como factor fundamental de la gestión institucional, y en consecuencia estar en la capacidad de tomar iniciativas innovadoras de autogestión financiera, fomentar el trabajo en equipo, generar redes con movimientos y flujo de información y comunicación para superar los desequilibrios organizativos, y sobre todo fomentar la participación de los actores intra y extra-universitarios en las decisiones.

En el mismo orden de ideas un informante recalco que: Prof.10. "Se debe estimular una cultura universitaria con base en acciones concretas a través de estrategias motivacionales que comprometan a la comunidad universitaria a desarrollar la fuerza académica de cada Escuela, de cada proyecto, de cada programa académico, mediante el fomento de prácticas sociales-colectivas."

\section{Conclusiones}

La gerencia universitaria, hoy en día se enfrenta a grandes retos que exigen el re-direccionamiento de sus funciones operativas y estratégicas hacia un enfoque más humano y colaborativo. Todo conlleva a que las universidades venezolanas, en el ejercicio constante de sus funciones, operaciones, procesos, puedan adecuar su visión en razón de su contexto y, en consecuencia, ejecutar sus estrategias incorporando el aspecto ético en todas la dimensiones de acción del gerente universitario como factor clave del éxito donde la justicia y la equidad sea el punto de apoyo de gestión y toma de decisiones.

Esta investigación conlleva a reflexionar sobre el concepto de gerencia universitaria como servicio, y obliga necesariamente a revisar la visión y misión las cuales tienen que ver con la interpretación de la razón de la institución y las relaciones y compromisos de la universidad con la sociedad. Esto incide de manera clara en el ethos académico, en la identidad institucional, en los conceptos de autonomía y democracia institucional y en los sistemas de valores colectivos e individuales de los actores del hecho académico frente a los compromisos y relaciones con el entorno.

Es por ello que la redimensión de los perfiles en las universidades autónomas debe comenzar con una decodificación de las relaciones que se establecen entre los miembros de la comunidad universitaria, como las establecen y cuáles son los resultados de estas acciones, 
Categorías de la acción gerencial universitaria autónoma

Rondón García, Yoselina

para posteriormente incitar a asumir como compromiso la concepción del poder como acción donadora de bienestar que no busca nada a cambio, ni establece un sentido de dominación, sino que es una acción dirigida a buscar la suprema felicidad del individuo.

También es de suma importancia asumir el reto de concientizar el pasado y reflexionar el presente como un proceso de reconocimiento de los paradigmas que han permeado las acciones de quienes tienen a su cargo la misión de garantizar la excelencia académica en el ámbito universitario como proceso de reflexión; lo cual permite sensibilizar las acciones que son emprendidas ya que estas, están sustentadas en la necesidad de la superación de un paradigma que ha impedido que prevalezca la academia sobre cualquier otro interés.

Es necesario, según lo demuestran los discursos vivos de los informantes, hacer cambios profundo en las formas de hacer gerencia en la universidad, es necesario erradicar el egocentrismo, el exceso de compromisos políticos partidistas que benefician a grupos minoritarios dentro de la Universidad e impiden el acceso a los derechos que toda la comunidad universitaria tiene por igual, se reclama la necesidad de darle prioridad a los intereses del colectivo sobre los intereses individuales, el rescate de la ética no considerada como valores objetivos sino altruistas que realmente establezca un compromiso y confianza genuino entre los actores que hacen vida en la ecología universitaria.

Es importante señalar que los actores entrevistados están condicionados por un contexto polarizado, dentro del cual cada quien asume la posición con la cual se identifica e interpreta la realidad desde esta lógica discursiva. Pero esta investigación lo que pretendió fue obtener percepciones futuristas orientadas al deber ser, y no al diagnóstico de la realidad actual de lo que perciben los informantes. La experiencia de las entrevistas arrojó, que independientemente de la posición política asumida por los actores, sus percepciones sobre una redefinición de la gerencia en la educación superior venezolana, coincidieron en su totalidad con una orientación humanística y ética.

El concepto de gerencia universitaria debe transformarse a una concepción más amplia y humana que abarque los siguientes elementos: La gerencia universitaria consiste en un proceso complejo en el cual está inmerso el actor clave de la gerencia con sus procesos psico-sociales, el contexto con su dinámica y realidad cambiante en la que se producen interrelaciones que se ven influenciados por factores psicológicos, antropológicos y sociológicos, incluso biológicos, que hacen de la acción gerencial una red compleja donde convergen un conjunto de manifestaciones, códigos de interpretación y valores que se entrelazan para poder establecer principios definitorios de la acción que motoriza la visión y misión de la comunidad universitaria basada en una conciencia histórica de sus propias realidades, en un reconocimiento del contenido espiritual de sus miembros, en una búsqueda constante de la felicidad del grupo y en un plan estratégico definido de hacia dónde quiere ir. 


\section{Referencias Bibliográficas}

Castells, Manuel (2009). Comunicación y Poder. Primera Edición. Editorial Alianza. España.

Declaración de la Conferencia Regional de Educación Superior en América Latina y el Caribe (CRES, 2008). Disponible en: http://www.sisbi.uba.ar/novedades/declaraciónCRES2008pdf. Consultado: 30/03/2011.

Fergusson, Alex (2003). El debate sobre la reforma universitaria. En: Revista La Universidad se Reforma III. Compilador Lanz Rigoberto. Orus. pp. 95116. Venezuela.

Lanz, Rigoberto (2005). En Verdad la Universidad no se reforma. Disponible en: www.voltairenet.org/9123931. Consultado el 03/03/2011.

Llanos-De la Hoz, Silvio (1997). Gestión Estratégica Universitaria. Revista Educación y Ciencias Humanas. Año V. Volumen 9. Decanato de Postgrado de la Universidad Nacional Experimental Simón Rodríguez. pp. 224227. Venezuela.
Martínez, Miguel. (2004). Ciencia y Arte en la Metodología Cualitativa. México: Editorial Trillas.

Méndez, Evaristo (2004). Cómo no Naufragar en la Era de la Información. Epistemología para Investigadores e Internautas. Ediluz. Zulia.

Ministerio de Planificación y Desarrollo (2007). Plan de Desarrollo Económico y Social de la Nación 2007-2013. Ministerio del Poder Popular para la Comunicación y la Información. Disponible en: http://www.cenditel.gob.ve/node/343.

Último: Consultado el 05/05/2010

Morin, Edgar (1999). La cabeza bien puesta. Repensar la Reforma-reformar el pensamiento. Ediciones Nueva Visión. Argentina.

Muros, Xiomara (2004). La Gerencia Universitaria. Ediciones OPSU. Venezuela.

Straus, Anselm y Corbin, Juliet (2002). Bases de la Investigación Cualitativa. Técnicas y Procedimientos para Desarrollar la Teoría Fundamentada. $\mathrm{N}^{\circ} 34$. Universidad de Antioquia. p. 11. Colombia.

Tapscott, Don (1998). Creciendo en un Entorno Digital. McGrawHill. España.

Valles, Miguel (1999). Técnicas Cualitativas de Investigación Social. Editorial Síntesis. España. 